\title{
Prospectiva 2025 de la Carrera de Ingeniería Química en algunos Países pertenecientes a la Organización de Estados Americanos (OEA)
}

\author{
Carmen C. Ramírez ${ }^{(1)}$, Jhon W. Zartha ${ }^{(2)}$, Bibiana Arango ${ }^{(3)}$ y Gina L. Orozco ${ }^{(2)}$ \\ (1) Universidad Pontificia Bolivariana, Escuela de Ingenierías, Facultad de Ingeniería Química, Circular \\ 1a. 70-01 Medellín, Antioquia- Colombia (e-mail: cecilia.ramirez@upb.edu.co). \\ (2) Universidad Pontificia Bolivariana, Escuela de Ingenierías, Facultad de Ingeniería Agroindustrial, \\ Circular 1a.70-01 Medellín, Antioquia- Colombia. (e-mail: jhon.zartha@upb.edu.co; \\ gina.orozco@upb.edu.co) \\ (3) Universidad Pontificia Bolivariana, Escuela de Ingenierías, Facultad de Ingeniería Industrial, Circular \\ 1a.70-01 Medellín, Antioquia- Colombia. (e-mail: bibiana.arango@upb.edu.co).
}

Recibido Abr. 12, 2016; Aceptado Jun. 13, 2016; Versión final Ago. 4, 2016, Publicado Dic. 2016

\begin{abstract}
Resumen
En el marco de la iniciativa Ingeniería para las Américas (EFTA, Engineering for the Americas), programa que establece las necesidades de mejoramiento y modernización de la enseñanza de la ingeniería en los países miembros de la Organización de Estados Americanos (OEA), se llevó a cabo el estudio prospectivo de la Ingeniería Química al 2025. El estudio tiene como objetivo el identificar y priorizar nuevas tendencias, temas, tecnologías, criterios de calidad para la Ingeniería Química para el año 2025. El estudio prospectivo fue realizado consultando a expertos nacionales e internacionales a través de tres rondas Delphi, por medio de un documento digital enviado por correo electrónico. El estudio se centró en el análisis de siete áreas medulares de la ingeniería química: tecnologías, líneas de formación, ingeniería de sistemas de procesos, fenómenos de transporte y operaciones unitarias, fisicoquímica y termodinámica, química e ingeniería de las reacciones y criterios de calidad para la educación en ingeniería química. El estudio arrojó en total 85 temas prioritarios
\end{abstract}

\section{Prospective 2025 of the Chemical Engineering Career in some Countries belonging to the Organization of American States (OAS)}

\begin{abstract}
In the framework of the Engineering for the Americas program (EFTA), that establishes the need for the quality improvement and modernization of engineering education in member countries of the Organization of American States (OAS), a prospective study of chemical engineering by 2025 was performed. The objective of the study is identifying and prioritizing new tendencies, topics, technologies and quality criteria for the chemical engineering profession. The prospective study was done consulting national and international experts through three Delphi rounds by submitting a document via email. The study focused on analysing seven core areas of chemical engineering: technologies, formation lines, systems process engineering, transportation phenomena and unit operations, physical chemistry and thermodynamics, chemistry and reaction engineering, quality criteria for education in chemical engineering. The study covered 85 priority issues.
\end{abstract}

Keywords: foresight; chemical engineering; Delphi method; quality criteria; new trend. 


\section{INTRODUCCIÓN}

La Ingeniería Química y sus industrias relacionadas (Charpentier, 2005), enfrentan, desde el punto de vista tecnológico y científico varios desafíos, que desencadenan en un reto a los campos de acción del ingeniero químico, orientándolos hacia las áreas de los nuevos materiales, la investigación de procesos innovadores para pasar de la química intermedia tradicional a nuevas especialidades, de igual forma, hacia la química del material activo e industrias afines, la búsqueda de nuevas fuentes de energía y la conservación y uso racional de las que actualmente posee, la biotecnología, la ciencia de las superficies, entre otras.

Los estudios relativos al futuro de la industria química indican que ésta juega un rol decisivo en la economía mundial. Los avances en los servicios de salud, los nuevos principios activos de la industria farmacéutica y la introducción de tecnologías que protegen el medio ambiente, son piezas claves que caracterizan el rol especial, pero a la vez de enorme responsabilidad, de este sector industrial. Hoy en día, los sectores más importantes son los productos agroquímicos, pinturas y barnices, gases industriales, productos de aseo personal y de limpieza, plásticos y polímeros, fibras sintéticas, industria farmacéutica, lo que en inglés se conoce como chemical specialties, y que podría traducirse como químicos especiales: "sustancias de moléculas altamente complejas que se sintetizan en varias etapas" (Charpentier, 2005.)

Garcés y Zartha (2009) citan el Informe de la Conferencia Mundial del Sector de Productos Químicos y Farmacéuticos (ICEM), emitido a fines de 2006, donde se proponen las tendencias de la industria química a nivel global, e indica que cada vez se invertirá más y se construirán nuevas plantas en las regiones del mundo que están creciendo económicamente, como los países árabes y Asia, y en particular, India y China, el creciente poder adquisitivo en estas regiones significa que mayores sectores de la población exigen bienes y servicios a los que antes no podían acceder. De igual forma, el gobierno chino en el doceavo plan a cinco años (2011-2015), establecen 7 estrategias industriales, 4 de ellas, estaban directamente relacionadas con el sector químico (Meyring, 2013) En este sentido, la Oficina Internacional del Trabajo, (OIT), también presenta un informe sectorial sobre la evolución de las fusiones y adquisiciones de la industria química y farmacéutica a nivel mundial, con indicadores sobre la reestructuración y los efectos en el empleo en dichas industrias. (Oficina Internacional del Trabajo, 2011).

Consecuentemente y ante estos retos se hace inevitable la evolución de la Ingeniería Química a través del tiempo, creando y desarrollando nuevas técnicas y herramientas para enfrentar estos cambios que se presentan en las industrias, lo que conlleva a que el campo de acción de la Ingeniería Química se amplíe cada vez más, por tal razón, los programas académicos y los grupos de investigación de las universidades, necesitan mantenerse actualizados, con miras a obtener un enriquecimiento teórico y práctico en conocimientos y habilidades que se adapten al nuevo orden económico y tecnológico mundial.

Fruto de la alianza entre la industria, egresados de ingeniería y la academia aparece en el 2004, una guía, para abordar la restructuración en la educación y la práctica en ingeniería, conocida como CDIO, bajo la premisa comúnmente aceptada, de que los graduados de ingeniería deberían ser capaces de concebir, diseñar, implementar y operar sistemas complejos de ingeniería, con valor agregado, en un ambiente moderno y basado en el trabajo en equipos para crear sistemas y productos (Conceive Desing Implement Operate, s.f.).

Las principales escuelas de ingeniería de Estados Unidos, Europa, Canadá, Reino Unido, África, Asia y Nueva Zelanda formaron esta iniciativa, como colaboración a nivel mundial para concebir y desarrollar una nueva visión de la enseñanza de la ingeniería, que identifica 73 elementos deseados en 4 grandes temas como: razonamiento y conocimiento técnico, competencias y atributos personales y profesionales, competencias interpersonales: trabajo en equipo y comunicación, concebir, diseñar, implementar y operar sistemas en la empresa y en contexto social.

En cuanto a las competencias que deberá portar un ingeniero químico, Crosthwaite et al. (2006), apoyado en el estudio realizado por el World Chemical Engineering Council, (2004) presenta las principales habilidades que necesita un ingeniero en el mundo laboral, e indica al sector educativo en ingeniería que debe centrar sus esfuerzos para garantizar el logro de estas dichas capacidades son: Capacidad para trabajar eficazmente en equipo, capacidad para analizar información, capacidad para comunicarse de manera efectiva, capacidad para recoger información y capacidad de autoaprendizaje.

\section{LA PROSPECTIVA}

En 1967, el francés Gastón Berger fue el primero en introducir la palabra prospectiva, entendida como el arte y/o la ciencia de estudiar y prever el futuro (Builes y Manrique 2000). Para Godet, (2000), la prospectiva es una reflexión para aclarar la acción del presente a la luz de futuros posibles, herramienta que permite 
organizar y estructurar de manera transparente y eficaz la reflexión colectiva sobre las apuestas y retos del futuro y la evaluación de opciones estratégicas. Dentro de las escuelas de prospectiva se pueden contar la escuela francesa y la escuela anglosajona, dentro de esta última el método Delphi se constituye en su principal método.

Alrededor de los años cincuenta, investigadores de la Rand Corporation, en Estados Unidos comenzaron a utilizar pronósticos tecnológicos, en el área de la producción para la defensa; de acuerdo con Escorsa y Valls (1997), fueron aquellos, quienes introdujeron el método de iteración con retroalimentación controlada conocido desde entonces como Delphi. Para 1970, los japoneses desarrollaron su propia estrategia de prospectiva, encaminada a prever el futuro en la ciencia y la tecnología, con un horizonte de tiempo de 30 años, este estudio se considera como el primer estudio de prospectiva tecnológica. Dentro de las primeras publicaciones más importantes sobre el método, aparece la de Linstone y Turoff (1975), en la década de los 70; desde entonces se ha utilizado en muchos países en diversos estudios prospectivos, siendo uno de los más usuales según Balaraman y Venkatakrishnan (1980), en la planeación educativa en diferentes niveles.

El método Delphi ha sufrido grandes cambios desde sus primeras aplicaciones, lo cual ha generado el nacimiento del concepto "Delphi modificado" el cual se caracteriza por el anonimato, la presentación de alternativas diferentes a los consensos y un número menor de rondas, entre otros aspectos. (Zartha, et. al 2015). (Zartha et. al 2013). El método presenta varias características fundamentales, la primera es, el anonimato, ningún experto conoce la identidad de los otros participantes que componen el grupo de discusión, lo que permite preservar la reserva de las respuestas y de los posibles cambios de opinión respecto a uno o varios de los temas tratados y elimina la influencia o el sesgo que puedan presentar diferentes expertos en la interacción personal. La segunda se refieren a la iteración y la respuesta estadística, que lo diferencian de otras técnicas grupales, lo cual se logra al presentar varias veces el mismo cuestionario, y gracias a elementos metodológicos, cada experto puede revisar sus planteamientos tomando como base los resultados de la fase anterior e ir modificando su opinión, de tal forma, que las respuestas de los demás participantes, obliga a reflexionar sobre la complejidad del tema en cuestión, lo que permite integrar de manera sistemática las opiniones del grupo, con un alto grado de consenso.

Un debate frecuentemente en este tipo de estudios es el que se refiere al número de expertos, Cabero y Barroso (2013) refiriéndose a Williams y Webb (1994); Powell (2003), expresan que "no hay consenso con respecto al número de expertos necesarios para llevar a cabo un estudio". De igual forma, encontraron diferentes propuestas, como las siguientes: Malla y Zabala (1978) que sugieren que su número debe oscilar entre 15 y 20; la de Gordon (1994) que lo sitúa entre 15-35; la de Landeta (2002) que indica que debe estar comprendido entre 7 y 30; García y Fernández (2008) para quienes el intervalo debe situarse entre 15 y 25.

Este trabajo contó con el apoyo del programa Ingeniería para las Américas EFTA-OEA., creado como un plan de acción, en respuesta de la primera reunión de Ministros y altas autoridades de ciencia y tecnología, en el marco del Consejo Interamericano para el Desarrollo Integral (CIDI) de la Organización de Estados Americanos (OEA), llevado a cabo en Lima, Perú en noviembre de 2004 (declaración de Lima, 2004). Esta iniciativa hemisférica busca "construir capacidades locales de ingeniería para crear conocimiento que asegure la solución de necesidades locales y abra puertas para competir por oportunidades globales" (Engineering for the Americas, 2008). Prevé además, el mejoramiento de la formación en ingeniería, la elaboración de mecanismos de aseguramiento de la calidad, la armonización de las modalidades del título profesional, el fomento de la innovación y el compromiso del Estado para respaldar la necesaria modernización de la educación en ingeniería y tecnología en la búsqueda de soluciones a problemas locales de cada país y de paso incrementar las oportunidades en la competencia global.

De igual forma, las estrategias definidas allí, buscan incrementar el número de egresados de los programas de ciencia, tecnología e ingeniería, la acreditación internacional de los programas, incrementar el número de mujeres en ingeniería, fortalecer el desarrollo de programas de excelencia y alianzas entre los sectores académicos, públicos y privados, incrementando la cooperación interamericana para la educación en áreas de ciencia, tecnología e ingeniería, identificar fortalezas y debilidades en innovaciones educativas, prácticas pedagógicas y curriculares, formas y tipos de graduación, asignaturas, prácticas exitosas, entre otros (Engineering for the Americas, 2008).

Dentro de este contexto, es necesario responder varias preguntas vitales para el liderazgo de las facultades y los departamentos de Ingeniería Química: ¿Qué capacidades se deben desarrollar en los futuros ingenieros químicos?, ¿Cuáles son las nuevas tendencias, variables de futuro, temas y tecnologías que deben ser incorporadas en los programas de Ingeniería Química para el año 2025?, ¿Qué criterios de calidad deben tenerse en cuenta en los programas de Ingeniería Química con miras al $2025 ?$ 


\section{METODOLOGÍA}

El estudio prospectivo se llevó a cabo a través de la metodología DELPHI a tres rondas, más específicamente de un Delphi normativo. (Linstone y Turoff, 1975). La finalidad del estudio fue identificar las tecnologías, temas y criterios de calidad que deben tener en cuenta los programas de Ingeniería Química de los países miembros de la OEA al 2025. Esta metodología se clasificó en cuatro etapas:

\section{Primera etapa exploratoria}

En esta etapa se escogió el grupo monitor, conformado por 4 magister en gestión tecnológica, un magister en automática y diseño, un PhD en tecnología de recursos forestales y un candidato a magister en gestión tecnológica, quienes realizaron la búsqueda de la información básica sobre las áreas medulares de la ingeniería química, que como ya se describió, es una disciplina de gran amplitud, luego éstas áreas fueron divididas en sub-áreas, y a su vez éstas en temas.

Los temas del área de las tecnologías fueron producto de consultas en artículos científicos de bases de datos especializadas en ingeniería, cruzando información con palabras claves como emergent technologies, future chemistry, chemical vision 2020, entre otros. Clark, (2009); Hieftje, (2009); Gray, (2009); Imperiali, (2009); Müller, (2009); Noyori, (2009); Tolfree y Smith, (2009).

De igual forma, las líneas de formación de la ingeniería química se extraen de artículos científicos como los presentados por Crosthwaite et al., (2006), Glavic, et al. (2009), Armstrong, (2006). Así mismo se hizo una revisión del estudio prospectivo de Ingeniería Química al 2019 presentado por Garcés y Zartha (2009) y otros estudios prospectivos realizados por el Grupo de Política y Gestión Tecnológica, para la Escuela de Ingenierías de la UPB. Zartha y Herrera, (2011). Para la elaboración del cuestionario en el tema de criterios de calidad para la educación en ingeniería, se tuvieron en cuenta tanto las capacidades que presenta la agencia acreditadora ABET, Accreditation Board for Engineering and Technology (1997), como las recomendaciones que presenta CDIO en Crawley et al., (2014).

En la tabla 1 se muestran las áreas medulares y las sub-áreas que dan un foco sobre el panorama de la ingeniería química del futuro y que a juicio del grupo monitor era importante conocer.

\section{Segunda etapa}

A la consulta, fueron convocados profesionales que se desempeñan como ingenieros químicos o de procesos, y que actúan en áreas afines y complementarias a la ingeniería química, pertenecientes a los diferentes sectores productivos, de servicios o académicos, de sectores públicos o privados y con diversos niveles de formación y de experiencia. La encuesta se aplicó además, a los expertos que asistieron a la sesión denominada OAS Engineering for the Americas, convocada por el evento LACCEI 2013 en México (Latin American and Caribbean Consortium of Engineering Institutions). De igual forma, la asamblea de la Unión Panamericana de Asociaciones de Ingeniería UPADI 2013 llevada a cabo en Medellín, y la reunión nacional de Directores de Ingeniería Química de Colombia en el Consejo Profesional de Ingeniería Química (CPIQ), fueron escenario de exposición del estudio con el propósito de adquirir otros expertos, a quienes posteriormente fue enviada la encuesta en formato electrónico, vía internet.

\section{Tercera etapa}

En la tercera etapa, se presentó el cuestionario de la segunda ronda Delphi, en la cual los miembros del panel deben reconsiderar sus estimaciones o respuestas y presentar sus respectivos argumentos. Se presentaron los temas por áreas y clasificados en temas prioritarios, en discusión y no prioritarios. Esta segunda ronda del ejercicio consistió en revisar los subtemas "en discusión" e identificar si alguno o algunos de ellos, en el concepto de los expertos, debe considerarse como prioritario, a cambio de alguno o algunos subtemas clasificados como tales.

\section{Cuarta etapa}

Para la cuarta etapa los expertos analizaron nuevamente sus respuestas, basados en la información recibida se tomaron sus decisiones finales y se envió el último cuestionario de la tercera ronda Delphi.

Para el cálculo del consenso logrado entre la primera y tercera ronda, se identificaron los participantes comunes en las tres rondas (29 en este caso) y con la información de la tercera ronda Delphi, se elaboró el informe final, con resultados definitivos, que representan el mayor nivel de consenso, el cual, según estudios realizados, a nivel mundial se considera óptimo entre el 50\% y el 100\%. (Builes y Manrique, 2000). 
Tabla 1. Áreas y sub-áreas

\begin{tabular}{|c|c|}
\hline Área & Sub-áreas \\
\hline 1. Tecnologías. & Sin sub-área \\
\hline \multirow{5}{*}{ 2. Líneas de formación } & Ciencias de los materiales \\
\hline & Materiales de la industria forestal \\
\hline & Química Ambiental \\
\hline & Biotecnología \\
\hline & Química de los alimentos \\
\hline \multirow{6}{*}{$\begin{array}{l}\text { 3. Ingeniería de sistemas de } \\
\text { procesos }\end{array}$} & Diseño de procesos \\
\hline & Control de procesos \\
\hline & Estadística y diseño de experimentos \\
\hline & Elementos de programación \\
\hline & Métodos numéricos para ingenieros de procesos \\
\hline & Balances en procesos de ingeniería química \\
\hline \multirow{6}{*}{$\begin{array}{l}\text { 4. Fenómenos de transporte } \\
\text { y operaciones unitarias }\end{array}$} & Fenómenos de transporte \\
\hline & Mecánica de fluidos para ingenieros químicos \\
\hline & Tecnología de partículas \\
\hline & Transferencia de calor \\
\hline & Transferencia masa \\
\hline & Operaciones de transferencia de masa \\
\hline \multirow{3}{*}{$\begin{array}{l}\text { 5. Fisicoquímica y } \\
\text { termodinámica }\end{array}$} & Fisicoquímica \\
\hline & Termodinámica Clásica \\
\hline & Termodinámica Química \\
\hline \multirow{4}{*}{$\begin{array}{l}\text { 6. Química e ingeniería de las } \\
\text { reacciones }\end{array}$} & Química orgánica \\
\hline & Bioquímica \\
\hline & Química analítica \\
\hline & Ingeniería de las reacciones químicas \\
\hline \multirow{9}{*}{$\begin{array}{l}\text { 7. Criterios de calidad para la } \\
\text { educación en ingeniería } \\
\text { química. }\end{array}$} & Atributos y competencias personales y profesionales \\
\hline & $\begin{array}{l}\text { Concebir, diseñar, implementar y operar sistemas en } \\
\text { el contexto social y la empresa }\end{array}$ \\
\hline & $\begin{array}{l}\text { Competencias a desarrollar en futuro ingeniero } \\
\text { químico }\end{array}$ \\
\hline & Métodos y estrategias de la enseñanza \\
\hline & Actividades extracurriculares \\
\hline & Presencialidad en el programa \\
\hline & Metodologías en laboratorios \\
\hline & Metodología en el trabajo autónomo del estudiante \\
\hline & Formación complementaria \\
\hline
\end{tabular}

\section{RESULTADOS}

En la figura 1 se presenta el nivel de formación de los expertos, el $62.5 \%$ del total de ellos, representa el sector académico y el $37.5 \%$ al sector productivo, tener representación de expertos en ambos sectores, es uno de los indicadores que permite equilibrio de enfoques en los resultados finales. El árbol temático se construyó lo suficientemente amplio para abarcar toda la disciplina, con un grado de especificidad tal, que permitiera tomar decisiones a mediano y largo plazo, lo que se convirtió en una característica sensible al estudio, por ser la ingeniería química una disciplina amplia y transversal a otras disciplinas, que implicaron una alta multiplicidad temática, para impedir el sesgo de los expertos, hacia algún campo de acción específico. En la tabla 2, se observan las 7 áreas principales y el número de temas correspondientes a cada una de ellas. 


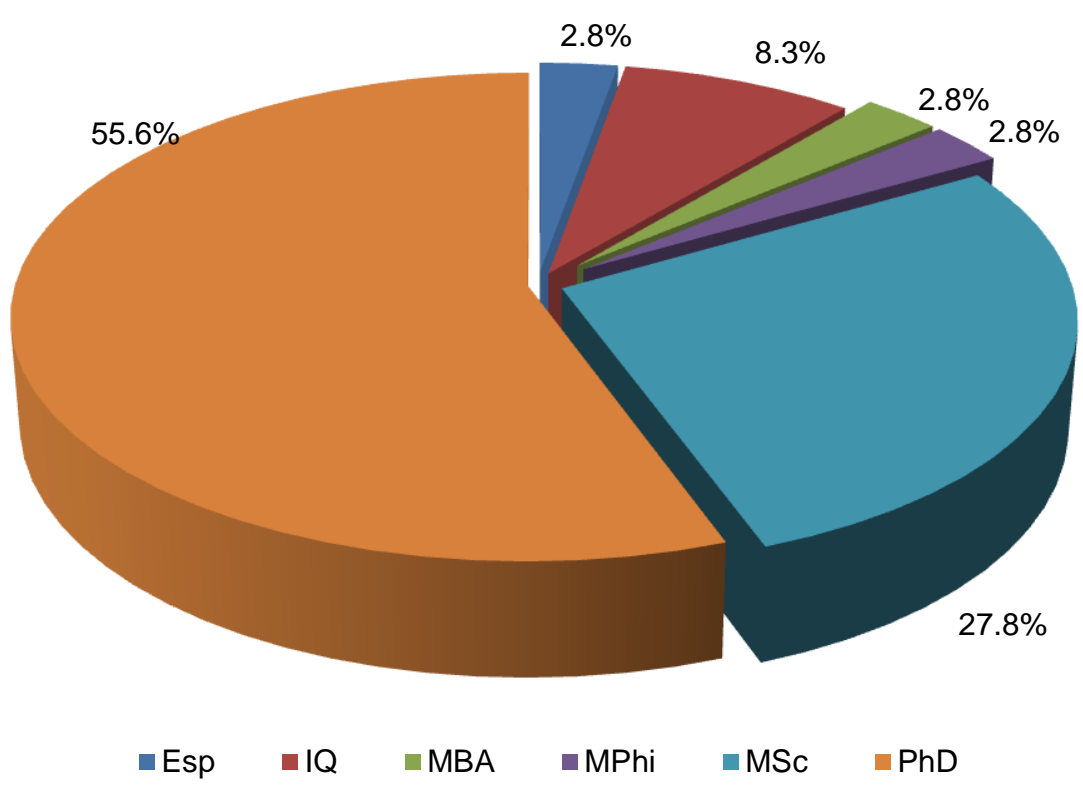

Fig. 1: Distribución de expertos por nivel de formación.

Tabla 2. Temas evaluados por área

\begin{tabular}{|l|c|}
\hline \multicolumn{1}{|c|}{ Área } & $\begin{array}{c}\text { Número de } \\
\text { Temas }\end{array}$ \\
\hline Tecnologías. & 17 \\
\hline Líneas de formación & 74 \\
\hline Ingeniería de sistemas de procesos & 42 \\
\hline Fenómenos de transporte y operaciones unitarias & 43 \\
\hline Fisicoquímica y termodinámica & 25 \\
\hline Química e ingeniería de las reacciones & 18 \\
\hline Criterios de calidad para la educación en ingeniería química. & 109 \\
\hline
\end{tabular}

\section{Primera ronda}

En esta etapa, el panel de expertos recibió el cuestionario elaborado para la primera ronda Delphi, se priorizaron los 328 temas que serían relevantes para la ingeniería química en el año 2025. La evaluación se realizó para cada tema en una escala de cero a cinco y la opción no sabe o no aplica y de forma independiente para cada una de las áreas. Luego de obtener las respuestas se procedió con el análisis estadístico que incluyó cálculos de moda, frecuencia modal, porcentaje de consenso y el valor promedio en los porcentajes de consenso, con esta información se estableció que un subtema era prioritario en la primera ronda si presentaba un valor modal mayor o igual a 4 en la calificación y un porcentaje de consenso superior al valor promedio en los porcentajes de consenso de su respectiva área. En la figura 2 se puede observar la representación del análisis de la información de la primera ronda de acuerdo a las respuestas de la primera ronda.

El área de mayor consenso entre los expertos es el área de "Criterios de calidad", con un $46.10 \%$ y la de menor consenso fue el área de tecnologías, con un 35.19\%; lo que es de esperarse debido a la diversidad en el perfil profesional de los expertos. En la tabla 3 se observan los promedios obtenidos en cada una de las áreas de Ingeniería Química provenientes de la primera ronda Delphi. 


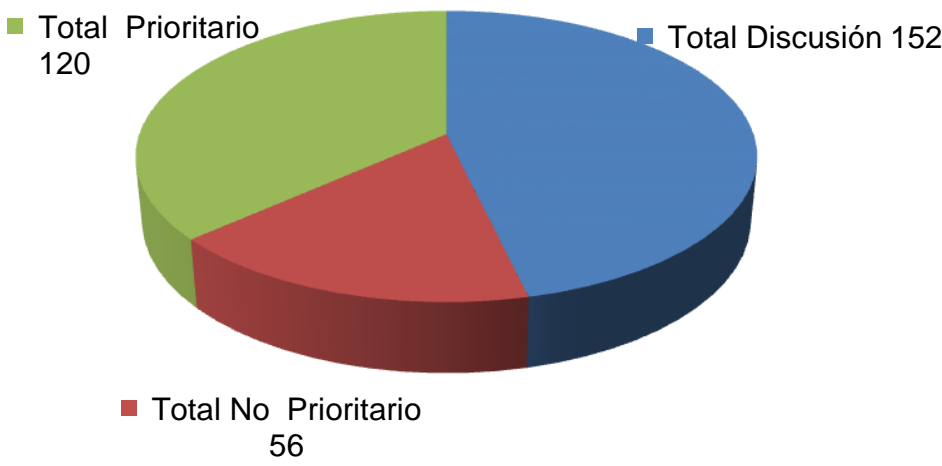

Fig. 2: Número de temas prioritarios, en discusión y no prioritarios obtenidos

Tabla 3. Resultados de la primera ronda con porcentajes de consensos por área.

\begin{tabular}{|l|c|}
\hline Área & Promedio (\%) \\
\hline Tecnologías. & 35.19. \\
\hline Líneas de formación & 36.92 \\
\hline Ingeniería de sistemas de procesos & 41.13 \\
\hline Fenómenos de transporte y operaciones unitarias & 39.24 \\
\hline Fisicoquímica y termodinámica & 41.74 \\
\hline Química e ingeniería de las reacciones & 38.11 \\
\hline Criterios de calidad para la educación en ingeniería química. & 46.10 \\
\hline
\end{tabular}

\section{Segunda ronda}

En esta etapa, se envió a los expertos el informe ejecutivo de la primera ronda, y se obtuvieron 29 respuestas de los 40 expertos que respondieron la primera ronda, lo que representa el $72.5 \%$. En esta oportunidad, cada experto cambió los temas que consideró mal clasificados, y justificó su decisión. Con los movimientos presentados en esta ronda, se realizó un conteo de los ítems que cambiaron (marcados con una " $x$ ") y los que no cambiaron, además se resaltaron los temas que contaron con alguna justificación. Los temas que no fueron cambiados, es decir, que su conteo de cambios fue cero, quedaban marcados como prioritarios para la tercera ronda, en total fueron 29 temas prioritarios que pasan a la tercera ronda demarcados con la letra $\mathrm{P}$, lo que informa al experto que es un tema ya fijo, información que se puede observar en la tabla 4.

A juicio de varios expertos, coinciden en bajar a discusión temas prioritarios tales como tecnologías en producción de hidrógeno, con argumentos como: la aplicación de las tecnologías en la producción de hidrógeno a corto y mediano plazo son incipientes, además, consideran que todavía es un tema muy exploratorio en el sector energético, de igual forma, indican que las tecnologías alimentarias y la bioquimiotecnología deben ser trabajadas por otras disciplinas de la ingeniería y no por la ingeniería química. Con respecto a los argumentos presentados en los subtemas en discusión del área de tecnologías, para que sean tenidos en cuenta como prioritarios, se destacan el uso de microreactores como una tendencia en la ingeniería química, que busca trabajar en condiciones más seguras y con procesos en los que se logre una mayor transferencia de masa y calor para llegar a procesos más efectivos, y el tema de las ciencias de las superficies, dado que la tendencia actual es hacia el diseño de productos y ésta se fundamenta en la modificación y manipulación de efectos de superficie.

\section{Tercera ronda y resultados finales}

Las respuestas de los 29 expertos que terminaron el estudio, generan el último análisis estadístico, el resultado final se construyó con base en: i) Identificación de los participantes comunes en las tres rondas (29 en este caso); ii) Con las calificaciones asignadas por los expertos a los subtemas en la primera ronda se calcula un valor modal de calificación. Si este valor modal es superior o igual a 4, este valor se mantendrá constante durante todas las rondas como prioritario; y iii) Se tomaron las calificaciones de la primera ronda como base para la tercera ronda. Los porcentajes de temas prioritarios definitivos por cada área, se muestran en la tabla 5. 
Tabla 4. Resultados de la segunda ronda, temas prioritarios.

\begin{tabular}{|c|c|}
\hline Área & Temas prioritarios \\
\hline \multirow{3}{*}{$\begin{array}{l}\text { Líneas de } \\
\text { formación }\end{array}$} & $\begin{array}{l}\text { Conversión de materiales complejos o peligrosos en productos reciclables o residuos } \\
\text { procesables. }\end{array}$ \\
\hline & Conservación, mejora y recuperación del medioambiente. \\
\hline & Procesos biotecnológicos. \\
\hline $\begin{array}{l}\text { Ingeniería de } \\
\text { diseño de } \\
\text { procesos }\end{array}$ & $\begin{array}{l}\text { Diseño de plantas flexibles, multiproducto, para procesos discontinuos (procesos batch). } \\
\text { Sistemas de varias fases. }\end{array}$ \\
\hline \multirow{8}{*}{$\begin{array}{l}\text { Fenómenos de } \\
\text { transporte y } \\
\text { operaciones } \\
\text { unitarias }\end{array}$} & Transporte en interfase y coeficientes de transferencia. \\
\hline & Balances macroscópicos en estado estable. \\
\hline & Balances microscópicos en estado estable. \\
\hline & Convección forzada. \\
\hline & Radiación. \\
\hline & Equipos de transferencia de calor. \\
\hline & Absorción. \\
\hline & Extracción. \\
\hline $\begin{array}{l}\text { Fisicoquímica y } \\
\text { Termodinámica }\end{array}$ & Leyes termodinámicas. \\
\hline \multirow{15}{*}{$\begin{array}{l}\text { Criterios de } \\
\text { calidad para } \\
\text { ingeniería }\end{array}$} & Demostrar capacidad para aplicar el conocimiento de las áreas profesionales de la ingeniería. \\
\hline & Pensar en forma lógica, conceptual, deductiva y crítica. \\
\hline & Tomar decisiones en presencia de incertidumbre. \\
\hline & Asume su mejoramiento personal y profesional a lo largo de toda la vida. \\
\hline & Escuchar activamente y mostrarse con empatía. \\
\hline & Liderar, dirigir personas, actividades, proyectos, empresas. \\
\hline & Trabajar en equipos y entornos internacionales. \\
\hline & Presentación oral y comunicación interpersonal. \\
\hline & Comunicación en idiomas extranjeros. \\
\hline & Conoce y comprende los problemas y asuntos contemporáneos. \\
\hline & Estudio y trabajos en equipo. \\
\hline & El uso de ambientes virtuales para el aprendizaje. \\
\hline & Metodologías en laboratorios: Aprendizaje basado en problemas. \\
\hline & Preparación de trabajos en grupo (seminarios, talleres, consultas). \\
\hline & Tutorías presenciales y virtuales. \\
\hline
\end{tabular}

Tabla 5. Resultados de la tercera ronda con porcentajes de consensos por área.

\begin{tabular}{|l|c|}
\hline \multicolumn{1}{|c|}{ Área } & $\begin{array}{c}\text { Porcentaje de Temas } \\
\text { Prioritarios (\%) }\end{array}$ \\
\hline Tecnologías. & 41.20 \\
\hline Líneas de formación & 24.32 \\
\hline Ingeniería de sistemas de procesos & 21.42 \\
\hline Fenómenos de transporte y operaciones unitarias & 23.25 \\
\hline Fisicoquímica y termodinámica & 32.00 \\
\hline Química e ingeniería de las reacciones & 38.90 \\
\hline Criterios de calidad para la educación en ingeniería química. & 23.80 \\
\hline
\end{tabular}




\section{CONCLUSIONES}

A partir del estudio prospectivo para la Ingeniería Química al 2025, se puede concluir:

1). El estudio arrojó en total 85 temas prioritarios, lo que representa el $25.9 \%$ del total de los temas (328 temas). De allí, 56 temas fueron resultado de la tercera ronda, lo que equivale al $65.9 \%$ de los temas prioritarios, que a su vez, se consideran como las respuestas mejor analizadas, debido, a que fueron aquellos temas con justificaciones o argumentos, que los expertos confirmaron como prioritarios, o que fueron rescatados de los temas en discusión en la tercera ronda. Los otros 29 temas prioritarios, son resultado de la segunda ronda y representan el $34.1 \%$ de los temas prioritarios.

2). A juicio de los expertos, los programas académicos de ingeniería química deben tener alta presencialidad en el programa ( $70 \%-100 \%)$, favorecer la clase magistral, al mismo tiempo, se sugiere el uso de ambientes virtuales por lo cual se hace necesario que cada programa establezca el porcentaje ideal para dar respuesta a los parámetros anteriores, sin perder el equilibrio entre la virtualidad, la experimentación y la presencialidad.

3). Las tecnologías prioritarias, tales como, tecnologías en materiales nanoestructurados, simulación molecular, materiales metálicos, ciencias de las superficies, pueden convertirse en un insumo para el establecimiento de nuevas líneas de investigación en grupos de investigación en el área, además, pueden servir para reforzar y validar los criterios de selección de las líneas de investigación actuales. De igual forma, los resultados del estudio también pueden servir de base para las actualizaciones curriculares, con el fin de generar estrategias de alineamiento con las tecnologías, las líneas de formación y aspectos de calidad que se obtuvieron en el estudio.

4). La modelación, simulación y optimización de los diferentes procesos, son identificados como prioritarios, tanto en el área de líneas de formación, como en el área de criterios de calidad para la enseñanza de la ingeniería, de modo que, se confirma que un ingeniero químico competitivo, debe tomar decisiones y dar respuesta a desafíos en tiempo real con ayuda de estas herramientas informáticas.

5). En relación con el conjunto de prioridades obtenidas, en los subtemas del área de criterios de calidad para la enseñanza de la ingeniería, constituyen un insumo de alta importancia, para el análisis y posterior definición del perfil de egreso de los programas de ingeniería química, según los sectores productivos y las necesidades de cada región donde sea necesario implementarlo.

6). Con respecto a la metodología se puede concluir que la herramienta para el método prospectivo, DELPHI utilizado en el desarrollo del estudio permite, establecer una plataforma de comparación y evaluación de diferentes temas con el fin de visualizar escenarios de futuro para el mejoramiento y el posicionamiento de las universidades a través de sus programas de ingeniería química.

\section{AGRADECIMIENTOS}

A la doctora Martha Beltrán y su equipo de la Organización de Estados Americanos, OEA -Sección de competitividad, tecnología e innovación, Departamento de desarrollo económico, Secretaría Ejecutiva para el Desarrollo Integral. Organization of American States - 1889 F Street N.W., Washington, D.C. Al director de la facultad de ingeniería química, de la Universidad Pontificia Bolivariana, Fabio Castrillón Hernández, por su compromiso con el estudio.

\section{REFERENCIAS}

Accreditation Board for Engineering and Technology. ABET. (en línea; http://www.abet.org/about-abet) (1997)

Armstrong, R. A vision of the curriculum of the future. Chemical Engineering Education, 40(3), 104-109 (2006)

Balaraman, S., y Venkatakrishnan, K. Identifying Engineering Education Goals and Priorities for the Future: an experiment with the Delphi Technique. Higher Education(9), 53-67 (1980)

Builes, C., y Manrique, J.. "Las prioridades investigativas en ingeniería mecánica: Un estudio prospectivo en Antioquia". Tesis de Magíster, Gestión Tecnológica, UPB -Medellín, Colombia (2000) 
Cabero, J., y Barroso, J. La utilización del juicio de experto para la evaluación de TIC : el coeficiente de competencia experta. Bordón Revista de Pedagogía, 65(2), 25-38 (2013)

Charpentier, J. Four main objectives for the future of chemical and process engineering mainly concerned by the science and technologies of new materials production. Chemical Engineering Journal(107), 3-17 (2005)

Clark, J. H. Chemistry goes green. Nature Chemistry, (en línea; http:// www.nature.com/naturechemistry) 1, 12-13 (2009)

Conceive Desing Implement Operate. CDIO. (en línea; http://www.cdio.org), (s.f.)

Crawley, E. F., Östlund, S., Brodeur, R, D., y Kristina, E. Rethinking engineering education: the CDIO Approach. New York: Springer (2014)

Crosthwaite, C., Cameron, I., Lant, P., y Litster, J. Balancing curriculum process and content in a project centered curriculum: in pursuit of graduate attributes. Education for Chemical Engineers, 1(1), 39-48 (2006)

Declaración de Lima (en línea; http://portal.oas.org/Portals/7/Ciencia_Tecnologia/documentos/Declaracion de Lima.pdf), (2004)

Engineering for the Americas. EFTA. (en línea; http://www.efta.oas.org), (2008)

Escorsa, P., y Valls, J. V. Manual de gestión e innovación tecnológica en la empresa. Santiago: CINDAAECI. p.116 (1997)

Garcés, B., y Zartha, J. W. Estudio prospectivo ingeniería química 2019. Producto №1. El contexto de la ingeniería química. Medellín: UPB. Escuela de Ingenierías (2009)

García, L., y Fernández, S. J. Procedimiento de aplicación del trabajo creativo en grupo de expertos. Ingeniería Energética, (en línea; http://www.redalyc.org/pdf/3291/329127758006.pdf) XXIX (2), 46-50 (2008)

Glavic, P., Lukmana, R., y Lozano R. Engineering education: environmental and chemical engineering or technology curricula - a European perspective. European Journal of Engineering Education. Vol. 34, No. 1, 47-61 (2009)

Godet, M. La caja de herramientas de la prospectiva estratégica. España: Librairie des Arts et Métiers (2000)

Gordon, T. J. The Delphi method. Futures Research Methodology (en línea; http://fpf.ueh.edu.vn/imgnews/04-Delphi.pdf) (1994)

Gray, H. B. Powering the planet with solar fuel. Nature Chemistry, (en línea; http://www.nature.com/nchem/journal/v1/n1/full/nchem.141.html) 1(7) (2009)

Hieftje, G. Weighing up the future of scientific tools. Nature Chemistry, (en línea; http://www.nature.com/nchem/journal/v1/n1/full/nchem.147.html) 1(10-11) (2009)

Imperiali, B. Interrogating biology with a chemical lexicon. Nature Chemistry, (en línea; http://www.nature.com/nchem/journal/v1/n1/full/nchem.144.html) 1(9-10) (2009)

Landeta, J. El método Delphi: una técnica de previsión del futuro. Barcelona: Ariel (2002)

Latin American and Caribbean Consortium of Engenieering Institutions. OAS Engineering for the Americas Encuentro. Prospective 2025: Chemical and Industrial Engineering, (en línea; http://www.laccei.org/LACCEI2013-Cancun/LACCEI2013.pdf), (2013)

Linstone, H. y Turoff, M. Delphi Method Technique and applications. Boston: Asdison-Wesley (1975)

Malla, F., y Zabala, I. La previsión del futuro en la empresa (III): el método Delphi. Estudios Empresariales (39), 13-24 (1978)

Meyring, N. China's chemical industry: the emergence of local champions. Reaction Magazine - Twelfth Edition, (en línea; https://assets.kpmg.com/content/dam/kpmg/pdf/2014/09/reaction-magazine-twelfthedition-v2.pdf) 2(16-39) (2013) 
Müller, A. Predicting a structured future. Nature Chemistry, (en línea;

http://www.nature.com/nchem/journal/v1/n1/full/nchem.140.html) 1(13-14) (2009)

Noyori, R. Synthesizing our future. Nature Chemistry, (en línea;

http://www.nature.com/nchem/journal/v1/n1/full/nchem.143.html), 1(5-6) (2009)

Oficina Internacional del Trabajo. Reestructuración, empleo y diálogo social en las empresas de las industrias química y farmacéutica. Ginebra: OIT, (en línea;

http://www.ilo.org/wcmsp5/groups/public/@ed_dialogue/@sector/documents/meetingdocument/wcms_16494 5.pdf), (2011)

Powell, C. The Delphi technique: myths and realities. Journal of Advanced Nursing, 41(4), 376-382 (2003)

Tolfree, D., y Smith, A. Roadmapping emergent technologies: planning the future, Troubador Publishing Ltd. (2009)

Williams, P., y Webb, C. The Delphi technique: A methodological discussion. Journal of Advanced Nursing (19), 180-186 (1994)

World Chemical Engineering Council WCEC How Does Chemical Engineering Education Meet the Requirements of Employment? (En línea; http://www.chemengworld.org/chemengworld_media/short_reportp-36.pdf), (2004)

Zartha S, J. W; y Herrera V, J. F. Prospectiva tecnológica: aplicación del método delphi en facultades y programas de ingeniería. Caso Universidad Pontificia Bolivariana. Medellín. UPB (2011)

Zartha S, J.W; Arango A, B; Vélez S, F.M; Coy M, A; Méndez N, K; Orozco M, G.L; Ávalos P, A.F; Durán, J; Beltrán M, M; Ríos J, M. Estudio de prospectiva de la ingeniería industrial al 2025 en algunos paises miembros de la OEA (2013)

Zartha S, J.W; Montes, J.M; Vargas M, E.E; Arias L, O; Hoyos C, J.L. El Método Delphi modificado. Un acercamiento desde la Metodología de Sistemas Suaves. Revista espacios. Vol. 36 (№ 17) Pág. 11 (2015) 
